\title{
Risk-Based Management of Overloads Caused by Power Injection Uncertainties Using Power Flow Controlling Devices
}

\author{
Priyanko Guha Thakurta, Member, IEEE, Ronnie Belmans, Fellow, IEEE, and \\ Dirk Van Hertem, Senior Member, IEEE
}

\begin{abstract}
Increased penetration of renewable energy leads to increased challenges for the transmission system operators (TSOs) to operate their system in a secure way. Through the limited means available to the TSOs to manage power flows in the system, reliability is jeopardized. Reliability must be managed differently, replacing the current ways of reliability management which fails as uncertainty increases. In this paper, a novel risk-based approach of system operation is proposed that can be helpful for the TSOs to assess the confidence of system operation day-ahead, that is, the probability of the forecasted system to end up in an insecure state is calculated. This paper only focuses on violation of power flow constraints in the system. It also demonstrates the increase of this operation confidence using already installed power flow controlling devices. It is shown that these devices aid in enhancing confidence of system operation by shifting power flows to a more optimal one in the light of generation uncertainty. The main emphasis is laid on preventive action. The proposed approach is demonstrated on test systems.
\end{abstract}

Index Terms-Optimal power flow (OPF), OPF under uncertainty, phase shifting transformers, risk management, stochastic optimization, transmission system operator (TSO) operation.

\section{NOMENCLATURE}

$t$

$N$

$B$

$c$

$N_{h}$

G

$W$

$T$

$\phi$

$\epsilon_{R}, \epsilon_{L}$
Time index.

Number of lines in the system.

Number of buses in the system.

Number of contingencies in the system.

Number of hours.

Set of conventional generator buses

Set of wind generator buses

Set of PFC devices

Standard normal cumulative distribution

function.

Allowed risks for right (R) and left (L) tail.
Manuscript received March 17, 2014; revised August 03, 2014, November 17, 2014, and December 28, 2014; accepted January 01, 2015. The KU Leuven Electa division is a founding member of Energyville. Paper no. TPWRS-003782014.

The authors are with the Electrical Engineering Department, Division Electa, KU Leuven, 3001 Heverlee, Belgium (e-mail: priyanko. guhathakurta@esat.kuleuven.be; ronnie.belmans@esat.kuleuven.be; dirk.vanhertem@esat.kuleuven.be).

Color versions of one or more of the figures in this paper are available online at http://ieeexplore.ieee.org.

Digital Object Identifier 10.1109/TPWRS.2015.2388444
$F \in \mathbb{R}^{N}$
Line flow vector.
$\bar{F} \in \mathbb{R}^{N}$
Upper line flow limits vector.
$F \in \mathbb{R}^{N}$
Lower line flow limits vector.
$D \in \mathbb{R}^{B}$
Demand vector.
$P_{i n j}$
Power injection vector.
$P_{i n j}^{\star} \in \mathbb{R}^{B}$
Basecase power injection vector.
$P_{G} \in \mathbb{R}^{\mid G}$
Vector of committed injections of conventional generators.
$P_{W}^{\star} \in \mathbb{R}^{|W|} \quad$ Forecasted wind injection vector.
$\sigma_{W}^{2} \in \mathbb{R}^{|W|} \quad$ Vector of variance of wind injections.
$u_{P F C} \in \mathbb{R}^{|T|}$ Vector of PFC device control settings.
$\bar{u}_{P F C} \in \mathbb{R}^{|T|}$ Vector of upper bound of PFC control settings.
$\underline{u}_{P F C} \in \mathbb{R}^{|T|}$ Vector of lower bound of PFC control settings.

\section{INTRODUCTION}

$\mathbf{U}$ NCERTAINTY in generation injection is increasing rapidly through the increased presence of renewable energy sources. The secure operation of such a system is increasingly difficult as power flows throughout the system vary constantly and forecast errors cause additional uncertainty. The operation of power systems is typically managed in a scheduling process which takes place from one day-ahead to real time. During this time frame, the uncertainty diminishes as one approaches real time. At the same time, the control means available to the operator reduce as well (or become considerably more expensive). In the presence of increasing uncertainties in the system due to intermittent generation sources, the day-ahead security scheduling/management is becoming a challenge for the transmission system operators (TSOs) in Europe. Previous literatures addressed this challenge extensively and proposed solutions accordingly. Panciatici et al. addressed this challenge in [1] and highlighted that a deterministic approach forecasting a single best guess of the operating point of the system for the next day or hours becomes inappropriate. This is mainly due to the fact that the real time scenario can be significantly different than the forecasted one. The TSOs must be prepared for such different types of uncertain scenarios while making their day-ahead security planning. One of the many challenges in this is the identification of such scenarios. In this context, the authors proposed a multi-stage decision making process under uncertainties and unified framework comprising of optimization algorithms for large scale power systems in an abstract 
manner that includes slow strategic preventive actions (e.g., starting up generation source, postponing maintenance works), fast preventive actions (e.g., generation re-dispatch) and corrective actions (e.g., topology changes, generation re-dispatch, changing settings of controllable devices). The authors also proposed ways to reduce the computational complexity of these huge scale problems to more solvable ones. However, choosing uncertainty sets, which is one of the many challenges in power system operational planning, has not been addressed in this paper. Reference [2] proposed an algorithm for the computation of strategic day-ahead control decisions in order to cover the worst cases that cannot be satisfied by best preventive or corrective actions. In this context, an optimization framework is developed to deal power system scheduling/planning under uncertainties in day-ahead over a set of power injection scenarios that may occur in the next day with appropriate preventive and corrective actions. The developed algorithm is highly computationally expensive due to significant number of uncertain scenarios to be considered. A methodology is also developed to determine the worst patterns of uncertain variables associated with each contingency [3]. Prime attention is laid on the adequate handling of decision variables arising from the operating rules of TSOs. The results showed that operation of controllable devices are triggered for some worst cases that are characterized by the interactions of uncertainties and maximum flow limits on the lines. The methodology has been tested on a very large scale network comprising of 9241 buses/nodes with 5126 contingencies.

In the light of these uncertainties, a flexible power system operation is required in order to maintain security and reliability of the system. Previously, the power system operation was quite straightforward as the generation was dominated mainly by conventional generators. More and more integration of intermittent generation will pose serious limitations to the current approach of power system operation and risk based approaches need to be developed in order to deal with uncertainties while maintaining low cost [4]. In other words, probabilistic methods need to be developed.

Traditionally, optimal power flow (OPF) is used in power system operation. This tool is helpful in determining the optimal set points of different control variables to keep the state variables of the system within bounds. An extended version of this tool caters the inclusion of contingencies which need to be taken into account, named as security constrained OPF (SCOPF) [1]-[3], [5], enabling preventive and corrective actions. In order to apply SCOPF to large scale power systems several methods have been proposed for efficient solving [6], [7] and contingency screening [8]-[11]. However, these tools are deterministic in nature.

The probabilistic approach to the load flow studies was first proposed by Borkowska for evaluation of power flow under load uncertainties [12]. Similar studies are performed considering wind generation [13] and branch outages [14]. Probabilistic methods have also been incorporated in SCOPF in many literatures. Vrakopoulou et al. proposed both DC [15] and AC [16] variants of probabilistic SCOPF to minimize day-ahead N -1 secure dispatch cost. Several literatures also addressed the unit commitment and economic dispatch problems in presence of uncertain variables [17]-[24].
Power flow controlling devices (PFCs) such as phase shifting transformers (PSTs) and high voltage direct current (HVDC) have gained increasing attention and application in the power system. Several of these devices are installed in the European power system, with many of them in a meshed system. These devices offer a solution which increases transmission capacity while avoiding the construction of new energy corridors [25].

Very few literatures considered these devices within the probabilistic framework. The work in [15] is extended in [26] to incorporate corrective control action with HVDC lines. The authors considered minimizing generation costs as the objective within the probabilistic SCOPF formulation. SCOPF formulations have also been addressed considering uncertainty, risk and controllability (using HVDC) in single and multi-area systems [27]. The authors in [28] addressed the challenges of solving risk-based SCOPF model with a new expression of severity function and a nested Benders decomposition with multi-layer linear programming method.

All the available literatures within the probabilistic framework considered generation dispatch as the objective, which comes at a high cost to the TSOs in Europe after decentralization. To the best of our knowledge, no prior work has been done to use PFCs in order to increase system operation confidence in the day-ahead operational planning. These devices are owned by TSOs and their operation comes at a near zero cost to them, thereby avoiding costly generation re-dispatch.

This paper addresses a risk-based optimal operation of power systems with high uncertainty from a TSO point of view, without considering generation re-dispatch. The contribution is three-fold.

1) A novel approach is developed in order to assess the confidence level that can be achieved in day-ahead operational planning for the TSOs taking power injection uncertainties into account. In other words, quantification of the amount of risk that the system can have is developed in this paper. In this way, the probability of system failure is visible to the operator.

2) A method is developed to increase the confidence level with the help of PFCs, such as PSTs and HVDC, that are already installed in the system, by shifting to a more optimal point considering uncertainties.

3) A method is developed to minimize the operator interventions for changing the control settings of the PFCs in a multiple hour time-scale for a predetermined confidence level of the whole system.

The proposed methodology can be applied to any kind of uncertainties, but the focus is laid on wind uncertainty in this paper. Moreover, the main focus in this paper is laid on the violation of transmission flow constraints in the system.

The paper is organized as follows. Section II describes the TSO operation under uncertainty. Section III describes the mathematical formulation of the addressed problem (corresponds to items 1 and 2 mentioned above). The proposed methodology is applied on three test systems and the results are shown in Section IV. Section V extends the formulated problem to multiple hour time-frame and addressed the operator concerns for their intervention in changing control settings of PFCs every hour. A methodology to minimize such hourly 


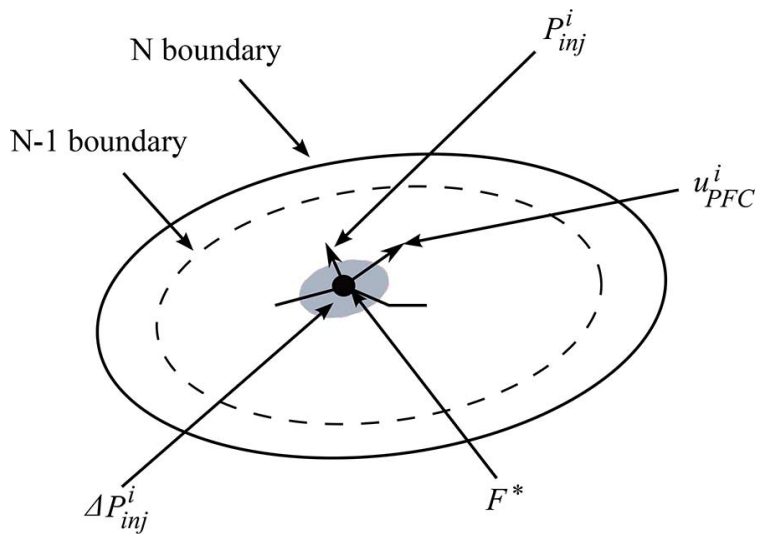

Fig. 1. Boundaries and influences in line flow space.

interventions is also proposed (corresponds to item 3 above). Finally Section VI draws the conclusions of the paper.

\section{OPERATION UNDER UNCERTAINTY FOR TSOS}

The security management under study is limited to power flows and thermal limits, without loosing generality of the addressed problem. The study of flexibility management in the transmission system is formulated in the $N$ dimensional space of linearized line flows. Linearized power flows have been considered as they are far easier to implement in optimization software, and yet yield sufficiently accurate results. A given solution of the power flow equations represents a point $\mathbf{F}$ in the vector space $\mathbb{R}^{N}$. A well-managed transmission system should be operated so that the flow does not exceed $\bar{F}$ on line flows:

$$
F\left(P_{i n j}^{\star}, \Delta P_{i n j}, u_{P F C}\right) \leq \bar{F}
$$

where both the power injection $P_{i n j}^{\star}$ with uncertainty $\Delta P_{i n j} \in$ $\mathbb{R}^{B}$ and the settings of PFC devices $u_{P F C}$ influence the flow $F$. As is depicted schematically in Fig. 1, line security constraints define boundaries associated with normal operation (solid line) and a single contingency (dashed line). The arrows represent flexibility control freedom $u_{P F C}$ while the circle represents the region of probable states due to uncertainty $\Delta P_{i}$ of power injections.

By linearization, sensitivity factors can be obtained that describe effects of changes in line flows due to changes in power injections and control settings of the PFC devices. At a chosen flow $F^{\star}$ (the basecase operating point considering committed injections at system buses, and that also include wind forecasts), the variations $\Delta P_{i n j}$ and $u_{P F C}$ produce a variation $\Delta F$ that can be expanded into component influences so that (1) becomes approximately

$$
\begin{aligned}
F= & F^{\star}\left(P_{i n j}^{\star}, u_{P F C}^{\star}\right)+\Delta F \\
\simeq & F^{\star}\left(P_{i n j}^{\star}, u_{P F C}^{\star}\right) \\
& +\underbrace{\left(\nabla_{P} F\right)}_{P T D F} \cdot \Delta P_{i n j}+\underbrace{\left(\nabla_{\left.u_{P F C} F\right)} F\right.}_{P F C D F} \cdot u_{P F C}
\end{aligned}
$$

where $\nabla_{P} F \in \mathbb{R}^{N \times B}$ and $\nabla_{u_{P F C}} F \in \mathbb{R}^{N \times|T|}$ represent sensitivities to changes in line flows due to changes in power injections (power transfer distribution factor or PTDF) and control

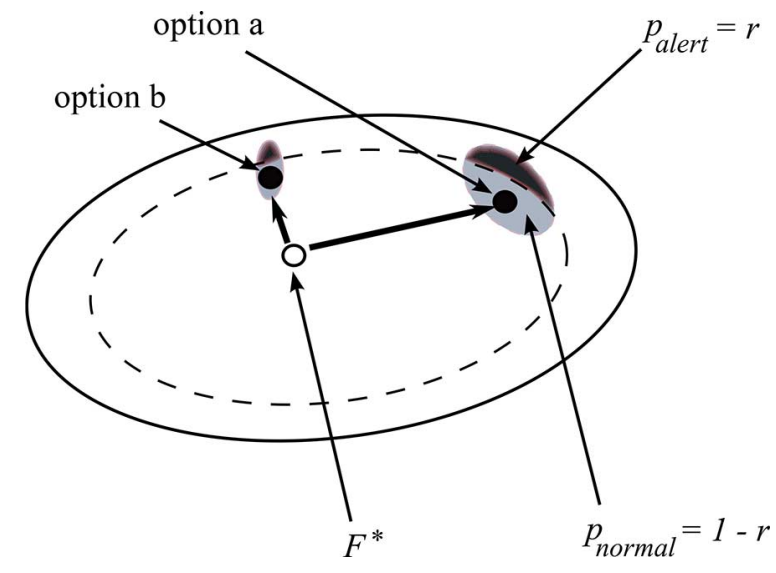

Fig. 2. Achieving boundary operation with designed risk level $r$.

settings (power flow controller distribution factor or PFCDF) ${ }^{1}$ of PFC, respectively.

Using this framework, it is possible to:

1) Quantify the improvement of transmission capacity by PFC devices in the presence of stochastic generation by shifting to one of a number of more optimal points. Fig. 2 shows that with the help of different available control options, which include PSTs, HVDC, demand side management, etc., the basecase operating point, which is based on forecasted intermittent generation and is a non-optimal state for most of the cases considering uncertainties, can be shifted to a state which includes the maximum uncertainty in intermittent generation without violating system operating constraints. The objective is that the operator can learn the optimal operation point, making maximum use of the system with a given confidence that the uncertainties present in the system will not cause overloads.

2) Quantify the effect of coordination of more than one PST in improving the transmission capacity in the presence of stochastic generation. The objective here is to show that the coordination of PFC devices among TSOs indeed helps in managing more uncertainties in their system.

The proposed methodology is tested both for $\mathrm{N}$ and $\mathrm{N}-1$ cases.

\section{Problem Approach AND FormUlation}

In this paper, the deterministic SCOPF is transformed to an explicit stochastic equivalent using chance constrained optimization in order to assess the confidence of a secure system operation. Chance constrained optimization is well known in the literatures [29]-[31]. The explicit stochastic optimization is superior to its implicit equivalent with respect to computational requirements, which is a severe limitation for large power systems.

The wind power in-feed can be mathematically written as a stochastic variable

$$
P_{W}=P_{W}^{\star}+\Delta P_{W}
$$

${ }^{1}$ Generalization of PTDF or phase shifter distribution factor (PSDF) for HVDC or PST influence, respectively 
where $P_{W} \in \mathbb{R}^{|W|}$. The vector $\Delta P_{W}$ consists of uncorrelated random variables which are assumed to be normally distributed with zero mean and standard deviation $\sigma_{W}$.

The power flows in the system change due to variations of wind in-feed. Hence the constraints on line flows can be written as

$$
\left[F=\left(\nabla_{P} F\right) \cdot P_{i n j}^{\star}+\left(\nabla_{P} F\right) \cdot \Delta P_{i n j}\right] \leq \bar{F}
$$

where $P_{i n j}^{\star}$ includes the committed injections of the conventional generators and the forecasted wind values $P_{W}^{\star}$ and $\Delta P_{i n j}$ includes the random wind variations with $\Delta P_{i n j_{i}}=0$ for $i \notin$ $W$. The random nature of $\Delta P_{i n j}$ restricts the use of this constraint. This constraint must be formulated probabilistically in the form of chance constraints [15], [16], [26], [27]:

$$
\mathbb{P}[F \leq \bar{F}] \geq 1-\epsilon
$$

where $\mathbb{P}$ represents the probability measure of the constraint that is to be realized with a minimum probability of $1-\epsilon$.

The authors in [15], [16], [26], and [27] solved such types of constraints using the scenario approach. In this approach, chance constraints are substituted with a finite number of hard constraints corresponding to different scenarios of the uncertainty vectors. The authors of [32] provide a bound on the number of scenarios that is needed to generate in order to achieve a probabilistic performance guarantees. This number grows linearly with respect to number of decision variables thereby leading to computationally expensive problems for systems of high dimensions. This approach is pretty unrealistic for day-ahead scheduling of a power system of a scale in Europe where there are considerable number of buses that contain intermittent generation.

In this work, the injections from wind energy are modeled as uncorrelated normally distributed random variables. The law of large numbers indicates that statistical smoothing effects due to the geographical dispersion of a large number of wind farms may result in the aggregate power approaching a normal distribution, as seen in studies such as [33]. Hence, the chance constraint (6) can be transformed to its analytical one, which is exact for uncorrelated normally distributed random variables [34]:

$$
\mathbb{P}\left[\frac{F-F^{\star}}{\sigma_{F}} \leq \frac{\bar{F}-F^{\star}}{\sigma_{F}}\right] \geq 1-\epsilon
$$

where $\left(F-F^{\star}\right) /\left(\sigma_{F}\right)$ represents a standard normal variate with a mean of zero and a variance of one. Hence, the stochastic chance-constraint is transformed into the following inequality:

$$
\phi\left(\frac{\bar{F}-F^{\star}}{\sigma_{F}}\right) \geq \phi\left(z_{1-\epsilon}\right)
$$

where $\phi\left(z_{1-\epsilon}\right)=1-\epsilon$. This yields the following nonlinear deterministic constraint:

$$
F^{\star}+z_{1-\epsilon} \sigma_{F} \leq \bar{F} .
$$

Different risks are obtained for the upper and lower bounds of the line flows, as shown in Fig. 3. Hence, the inequality constraint (9) can be divided into

$$
\begin{aligned}
& F^{\star}+z_{1-\epsilon_{R}} \sigma_{F} \leq \bar{F} \\
& F^{\star}-z_{1-\epsilon_{L}} \sigma_{F} \geq \underline{F} .
\end{aligned}
$$

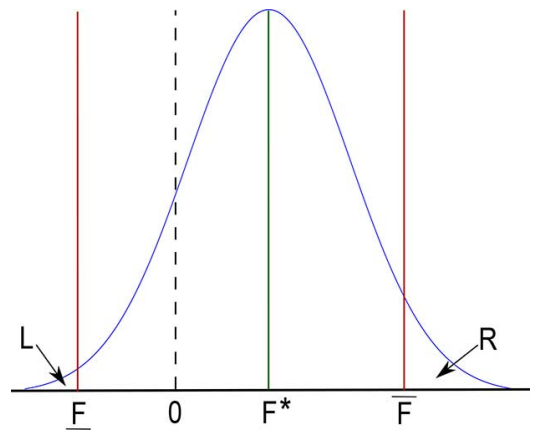

Fig. 3. Achieved confidence of a transmission line.

The variance on line flows $\sigma_{F}^{2}$ due to random uncorrelated wind injections is given by [12]

$$
\sigma_{F}^{2}=\left(\nabla_{P} F\right)^{2} \cdot \sigma_{W}^{2}
$$

Hence, the standard deviation $\sigma_{F}$ of line flows is given by

$$
\sigma_{F}=\sqrt{\left(\nabla_{P} F\right)^{2} \cdot \sigma_{W}^{2}} .
$$

In the available literatures [15], [16], [26], [27], the cost of generation dispatch is minimized as an objective, taking into account a predefined probability of violation of generation and line constraints. The probability violation is the same for all of the lines and that of the generators. In other words, the constraint (6) holds with a predefined probability, that is, $1-\epsilon$ is determined in advance. In this work, the main objective is to achieve a maximum overall system operation confidence, hence the product of the cumulative distribution function of each line needs to be maximized. In other words, $\epsilon$ is an optimization variable which needs to be minimized in order to achieve minimum overall system operation risk.

The formulated optimization problem is as follows:

$$
\max _{u_{P F C}} \prod_{N}\left[\phi\left(z_{1-\epsilon_{R}}\right)-\phi\left(z_{1-\epsilon_{L}}\right)\right]
$$

subject to

$$
\begin{aligned}
& F^{\star}=\left(\nabla_{P} F\right) \cdot\left(P_{G}+P_{W}^{\star}-D\right) \\
& F_{c}^{\star}=\left(\nabla_{P} F_{c}\right) \cdot\left(P_{G}+P_{W}^{\star}-D\right) \quad \forall c \\
& F^{\star}+z_{1-\epsilon_{R}} \sqrt{\left(\nabla_{P} F\right)^{2} \cdot \sigma_{W}^{2}}+\left(\nabla_{u_{P F C}}\right. \\
& F^{\star}+z_{1-\epsilon_{L}} \sqrt{\left(\nabla_{P} F\right)^{2} \cdot \sigma_{W}^{2}}+\left(\nabla_{u_{P F C}}\right. \\
& F_{c}^{\star}+\left(\nabla_{u_{P F C}} F_{C}\right) \cdot u_{P F C} \leq \bar{F} \quad \forall c \\
& F_{c}^{\star}+\left(\nabla_{u_{P F C}} F_{C}\right) \cdot u_{P F C} \geq \underline{F} \quad \forall c \\
& z_{1-\epsilon_{R}} \geq 0 \\
& z_{1-\epsilon_{L}} \leq 0 \\
& \underline{u}_{P F C} \leq u_{P F C} \leq \bar{u}_{P F C} .
\end{aligned}
$$$$
F^{\star}+z_{1-\epsilon_{R}} \sqrt{\left(\nabla_{P} F\right)^{2} \cdot \sigma_{W}^{2}}+\left(\nabla_{u_{P F C}} F\right) \cdot u_{P F C} \leq \bar{F}
$$$$
F^{\star}+z_{1-\epsilon_{L}} \sqrt{\left(\nabla_{P} F\right)^{2} \cdot \sigma_{W}^{2}}+\left(\nabla_{u_{P F C}} F\right) \cdot u_{P F C} \geq \underline{F}
$$

Constraint (15) represents the mean or basecase flows of the system and is the dot product of the PTDF matrix and the injection vector. In other words, this determines the basecase operating point of the system. Constraint (16) represents the flows on the lines for each contingency considered. Constraints (17) and (18) are the analytical reformulation of the flow constraints, 
using the properties of the Normal distribution. $\left(\nabla_{P} F\right)^{2}$ represents squaring individual elements of the PTDF matrix. Each constraints (17) and (18) have an individual probability of being violated, since $\left(\epsilon_{L}, \epsilon_{R}\right) \in \mathbb{R}^{N}$. Constraints (19) and (20) represent the security constraints or $\mathrm{N}-1$ constraints imposed on the system. The probit function must be positive and negative for the upper bound and the lower bound of the line flows respectively, as represented by constraints (21) and (22), respectively. Constraint (23) represents the upper and lower bounds of the PFC control settings. The objective is to maximize the overall system confidence represented by the product of individual confidences of each line flows, and is given by (14).

\section{ILLUSTRATIVE EXAMPLES}

The approach described in Section III is demonstrated on IEEE 14-bus [35], IEEE One Area RTS-96 [36], and 2736-bus Polish systems with installed PSTs. MATPOWER software [37] is used for the purpose.

\section{A. IEEE 14-Bus System}

The idea of quantification of transmission capacity increase in presence of stochastic generation with the help of PSTs, described in Section II, is dealt deeper in this system, as the line limits in this system are not defined. The single line diagram is shown in Appendix A. The proposed optimization problem is also validated from the results obtained by Monte Carlo (MC) simulation. The line limits of all the lines are very high except lines 1 (between buses 1-2) and 2 (between buses 1-5), which are $150 \mathrm{MW}$ each. Aggregated wind farms of installed capacities of $200 \mathrm{MW}$ and $300 \mathrm{MW}$ are connected at buses 2 and 3 of the system, respectively, and the total system load is increased twice as that of the given values. A PST is installed on line 2 of the system.

In the basecase scenario, the forecasted wind power in-feeds equal $50 \%$ and $67 \%$ of the corresponding installed wind capacities, with 100-MW and 200-MW in-feeds at buses 2 and 3, respectively. The forecast error $\sigma_{W}$ of the wind in-feeds at the buses is assumed to be $14 \%$ of the corresponding installed capacities. The deviations of the wind power in-feed from the forecasted value are compensated by the slack bus generator at bus 1. No contingencies are taken into account in this case. The PST angle is varied between \pm 30 degrees as a continuous variable to avoid additional computational cost. However, PST operates with discrete taps in practice. The actual tap setting can be found by selecting the nearest discrete tap positions.

Lines 1 and 2 are the centers of attraction as the line limits of these two lines are chosen such that they hit their limits. The probability of all other lines hitting their limits is 0 thereby having a confidence level of $100 \%$ for all these lines. The basecase operating point has $126.78 \mathrm{MW}$ and $87.45 \mathrm{MW}$ through lines 1 and 2, respectively.

Fig. 4 shows the results of the proposed approach. The confidence level for line 1 of the system without a PST is $71.2 \%$. The total system confidence in presence of PST is $92.47 \%$, with individual line confidences of lines 1 and 2 equal to $93.7 \%$ and $98.69 \%$, respectively. The obtained optimal PST angle from the proposed methodology is 7.85 degrees.

For MC simulation, 140 normally distributed points each for buses 2 and 3 are generated for the wind generation taking the

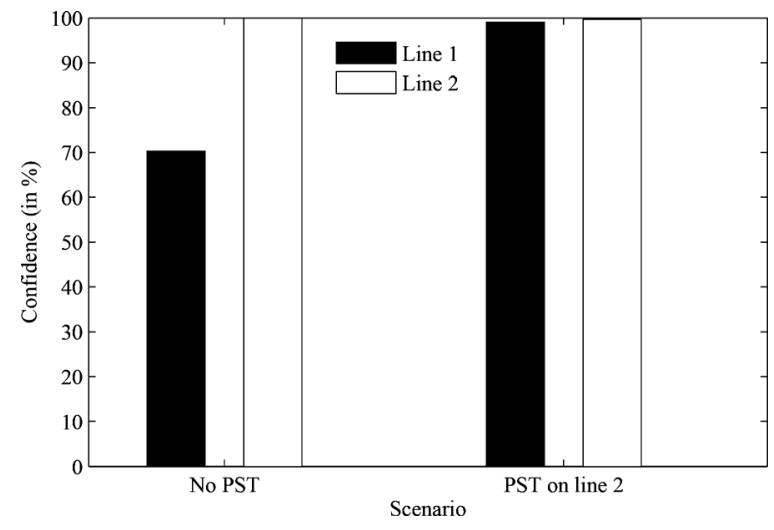

Fig. 4. Confidence level on selected lines for $\mathrm{N}$ of IEEE 14-bus system.

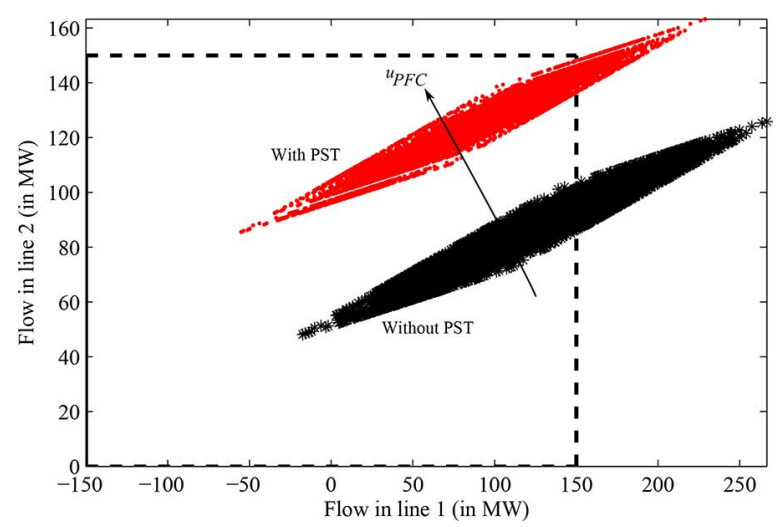

Fig. 5. Shifting of uncertainty cloud with PST installed on line 2.

above-said standard deviations into account. Each point for bus 2 is then combined with each point for bus 3 resulting in 19600 combined points (scenarios). A mixed integer linear programming (MILP) problem is formulated to push the cloud within the safe operating region with the help of PST. The MILP problem is given in Appendix C.

Fig. 5 shows the result of MC simulation. The dashed lines in the figure represents the secured region of operation for lines 1 and 2 . The negative limit of line 2 is not shown in the figure as it is not relevant in this case. As is evident from the figure, the uncertainty cloud is pushed to a more secured region with the help of the PST by diverting flows from line 1 to line 2 for maximum of the cases, thereby increasing system confidence in handling uncertainties. Without the PST the number of points outside of security boundary for line 1 is 5650 (represented by "+" dots in the figure), thereby posing a risk of $28.83 \%$ and hence a confidence of $71.17 \%$. In presence of the PST, the number of points out of bounds for line 1 is 1246 and that for line 2 is 239 (represented by "o" dots in the figure), leading to risks of $6.36 \%$ and $1.22 \%$, respectively, in turn having confidences of $93.64 \%$ and $98.78 \%$, respectively. Hence the total system confidences without and with PST are $71.17 \%$ and $92.5 \%$, respectively, which are similar to that obtained by the proposed methodology. The system at this stage is pushed to its limits reducing the risk of being overloaded. The optimal PST angle for this shift is 7.85 degrees, which is same as that obtained by the proposed methodology. 


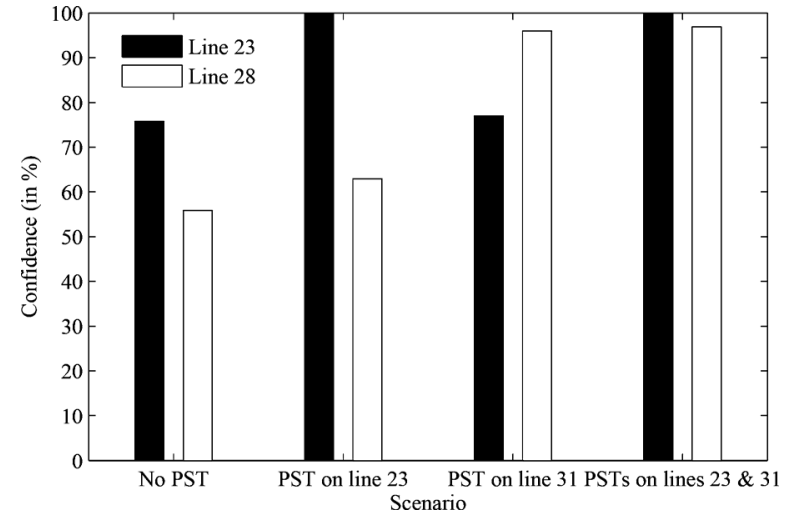

Fig. 6. Confidence level on selected lines for $\mathrm{N}$ of IEEE One Area RTS-96 system.

A careful observation of Fig. 5 reveals that there is still some margin left for line 1 to shift the cloud even further. Pushing the cloud further leads to the number of points out of bounds for line 1 and line 2 are 1015 and 644, respectively, leading to risks of $5.18 \%$ and $3.29 \%$, respectively. Hence the total system confidence amounts to $91.71 \%$ which is less than the one mentioned above. The optimal PST angle in this case is 9.05 degrees. Hence the system is operated at maximum achievable confidence with the previous PST setting.

The MILP problem stated above for MC simulation is solved using CPLEX solver of version 12.3.0. Moreover the proposed optimization problem is solved using KNITRO solver of version 7.0.0. Both the optimization problems are solved in an Intel Core 2 Quad, $2.83 \mathrm{GHz}$ with $4 \mathrm{~GB}$ of RAM on GAMS platform [38]. The solving time for the MILP is 50 min approximately, whereas that of the proposed approach is $32 \mathrm{~s}$ approximately.

\section{B. IEEE One Area RTS-96 System}

The RTS-96 system is a more realistic system for performing reliability studies, the single line diagram of which is given in Appendix B. Both $\mathrm{N}$ and $\mathrm{N}-1$ cases along with PST coordination are taken into account in this case. A wind farm of $500-\mathrm{MW}$ installed capacity is installed at bus 17 of the system. Two PSTs are installed on lines 23 (between buses 14 and 16) and 31 (between buses 17 and 22) of the system. The forecasted wind power in-feed equals $50 \%$ of the installed wind capacity, leading to $250 \mathrm{MW}$ at the bus. The forecast error $\sigma_{W}$ is assumed to be $14 \%$ of the installed wind capacity. Again, the deviations of the wind power in-feed from the forecasted value are compensated by the slack bus generator at bus 13 . Moreover, the committed generations of conventional generators at buses 1,2 , and 7 are reduced from $172 \mathrm{MW}, 172 \mathrm{MW}$ and $240 \mathrm{MW}$ to 100 $\mathrm{MW}, 50 \mathrm{MW}$ and $100 \mathrm{MW}$, respectively.

Fig. 6 shows the confidences for lines 23 and 28 (between buses 16 and 17) in the system for $\mathrm{N}$ situation. These lines are the bottlenecks for handling uncertainties in absence of the PSTs and hence are of central interest. The confidences for all other lines in the system are $100 \%$. The figure clearly reveals that the system can be operated with higher confidence with the help of PSTs. Another noticeable fact from the figure is the increase of system operation confidence if multiple PSTs are controlled in concert.

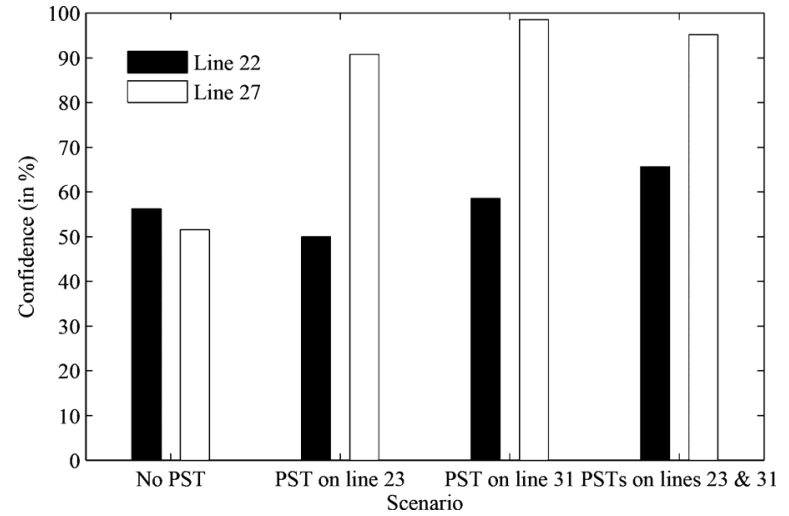

Fig. 7. Confidence level on selected lines of IEEE One Area RTS-96 system taking $\mathrm{N}-1$ constraints into account.

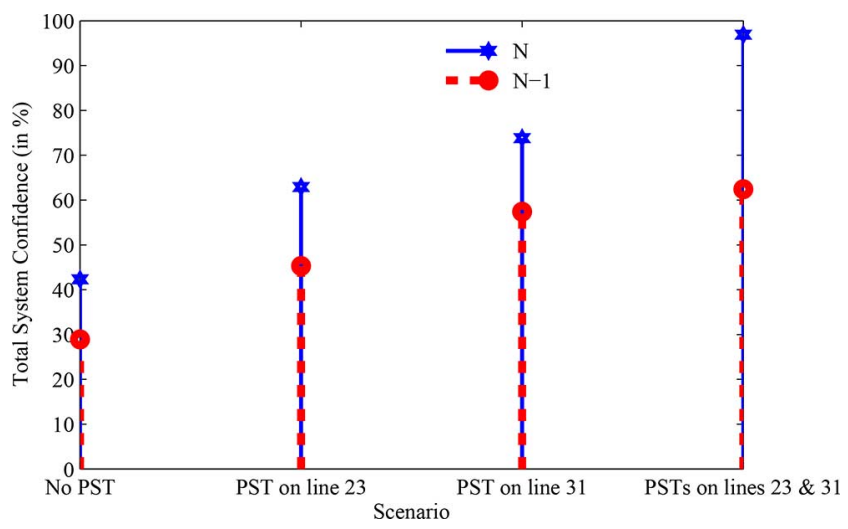

Fig. 8. Total system confidence for IEEE One Area RTS-96 system with wind in-feed.

In Fig. 2, it is clear that the feasible region of operation becomes less for $\mathrm{N}-1$ case as compared to $\mathrm{N}$ case. This leads to a lower system confidence level that can be achieved for $\mathrm{N}-1$ situation. To analyze this, line 6 (between buses 3 and 9) is considered a contingency and taken out of service. It is seen that the lines of interest for this case are 22 (between buses 13 and 23) and 27 (between buses 15 and 24) whereas all other lines have confidences of $100 \%$. Fig. 7 shows the individual line confidences for these two lines for the different scenarios. It is again evident that a PST is able to increase system operation confidence even for a contingency in the system. The same figure also reveals the effect of coordinated operation of PSTs in the system to increase the system confidence. Fig. 8 shows the achieved total system confidences for the different scenarios. The fact of having constrained feasible region for $\mathrm{N}-1$ is evident from the figure, since the dotted line representing the system confidence for $\mathrm{N}-1$ situation is systematically below the solid line representing the $\mathrm{N}$ situation. It is also evident that the coordinated operation of PSTs in a system significantly enhances the confidence of operating the system securely in face of uncertainties. This fact can be exploited by the TSOs in Europe to coordinate PST operations in their systems in order to minimize the risks due to uncertainties in their system.

During real-time operation, the renewable injections can be such that the system may end up in the risk zone $r$ (Fig. 2) during which the system operation becomes endangered. It is interesting to see whether the installed PFCs are able to bring 
TABLE I

SimUlation Time RESUltS ON LARGER SySTEMS

\begin{tabular}{|c|c|c|c|c|c|}
\hline \multirow[t]{2}{*}{ Simulated system } & \multicolumn{3}{|c|}{ Considerations } & \multicolumn{2}{|c|}{ Time for completion (seconds) (approx.) } \\
\hline & Number of contingencies & Number of uncertainties & Number of installed PSTs & MC & Proposed methodology \\
\hline IEEE 14-bus & None & 2 & 1 & 1826 & 0.199 \\
\hline IEEE RTS-96 & 1 & 1 & 2 & 1254 & 0.203 \\
\hline IEEE 118-bus & 10 & 5 & 3 & 72315 & 0.635 \\
\hline 2736-bus Polish system (summer peak) & 30 & 10 & 6 & Terminated without result & 385 \\
\hline
\end{tabular}



(a)

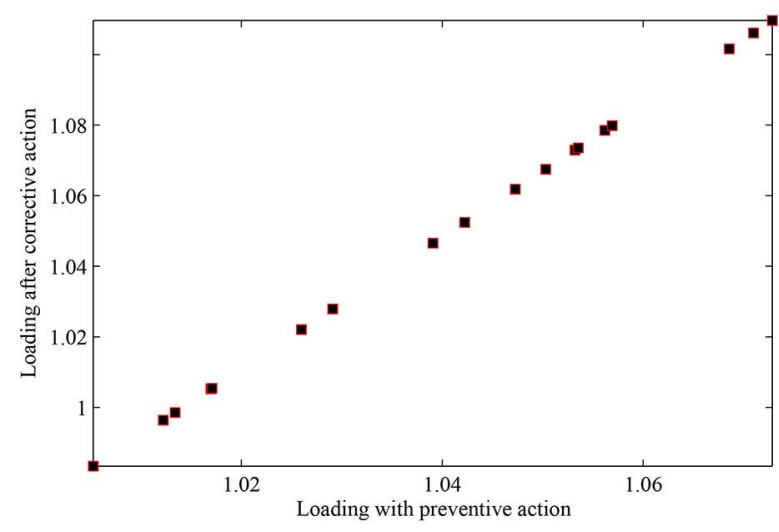

(b)

Fig. 9. Role of PFCs in corrective action when system ends at risk zone $r$ during real-time operation. (a) IEEE 14-bus system. (b) IEEE One Area RTS-96 system.



Fig. 10. Total system confidence for 2736-bus Polish system.

the system back to a secured state by respecting the system boundaries. In order to demonstrate this, such renewable injections are considered for which the flows of the system end up in the risk zone $r$ for both the above-mentioned systems at the corresponding renewable generation buses. The methodology proposed in section $\mathrm{CM}_{\mathrm{MO}}$ of [39] is used to bring the system back to the secured state from the risk zone. Fig. 9 shows the results for the system operated at the risk zone. The horizontal axis shows the loading of the system with the control setting of the PSTs fixed to the value obtained from optimization problem (14)-(23), whereas the vertical axis shows the loading of the system with a varying control settings between \pm 30 degrees. Loading is defined as the ratio of the line flow to its maximum permissible. Unity loading represents that the power flow through the line is at its maximum permissible. It is evident from Fig. 9(a) that the system can be brought back within the secured region for some of the states with the PST, whereas Fig. 9(b) shows that neither of the states can be made secure with coordinated control of the PSTs (considered for N situation). For such system states, other control means, such as generation re-dispatch, are required to make the system secure.

\section{2736-Bus Polish System}

The proposed methodology is also applied on this large system. The profile for the summer peak is considered. The total system load is increased by $2 \%$, the total conventional generation is reduced by $6 \%$ and the line limit for each line is increased by $10 \%$. The buses considered for uncertain injections are 17, 106, 115, 132, 145, 560, 807, 975, 1114, and 2539 with forecasted values of $200 \mathrm{MW}$ (buses 17, 106, 132, and 145), $100 \mathrm{MW}$ (buses 115, 1114, and 2539), $300 \mathrm{MW}$ (bus 560), $250 \mathrm{MW}$ (bus 807), and $150 \mathrm{MW}$ (bus 975). The forecast error $\sigma_{W}$ is assumed to be $60 \mathrm{MW}$ for all the buses. The deviations of the wind power in-feed are compensated by the slack bus generator at bus 28. 6 PSTs are installed on lines 18 (2727-67), 24 (2729-47), 83 (58-42), 359 (2584-349), 408 (2456-336), and 538 (381-239). Thirty contingencies are considered for simulations.

Fig. 10 shows the total system confidence for the Polish system considering different scenarios of PST operations. It is again evident that the total system confidence increases when all the PSTs are controlled in a coordinated manner.

The optimization problem for the Polish system consists of 20256 variables and 14017 constraints without any contingencies, whereas 125341 variables and 329283 constraints with 30 contingencies (both include all the PSTs).

Table I shows the simulated time of the proposed methodology against its MC variant for 4 types of systems. These simulations are performed on an Intel Xeon 3.3-GHz Windows server with $128 \mathrm{~GB}$ of installed RAM. However, the resources of this server are shared among many users. 


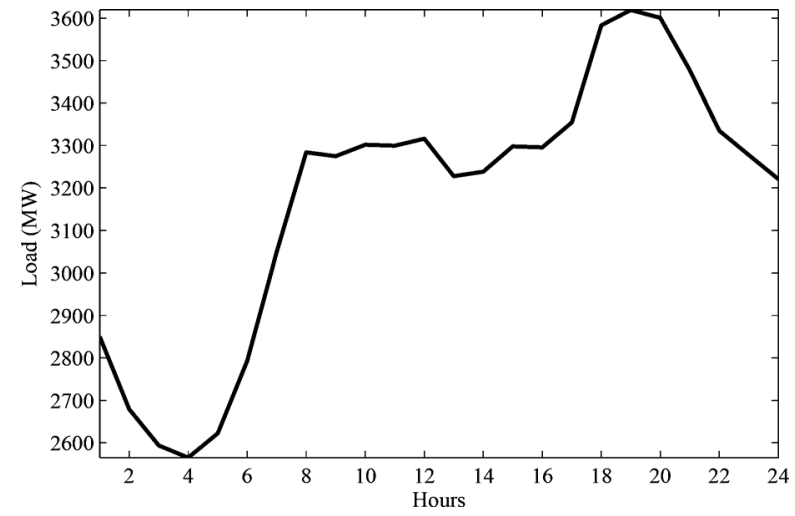

Fig. 11. Load profile.

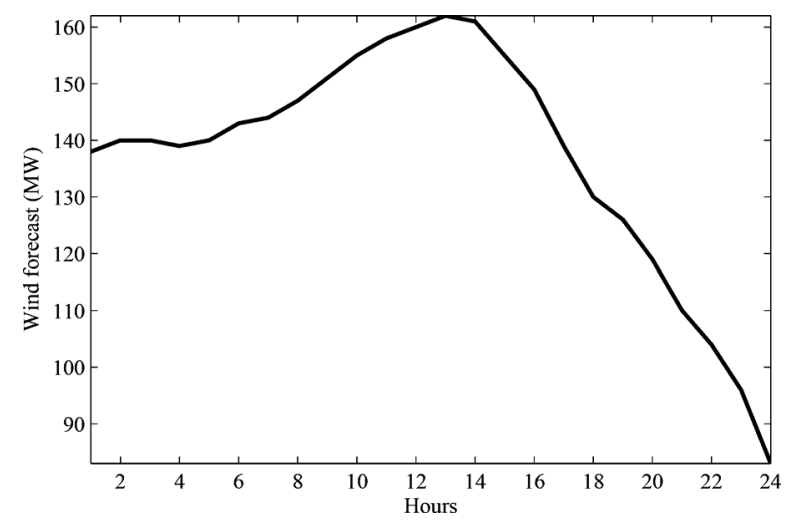

Fig. 12. Wind forecast for bus 17 .

It is evident that the proposed methodology can easily be implemented for large power systems.

\section{Extension of THE PROPOSED APPROACH TO Multiple Hour Time-ScAles}

The proposed methodology is demonstrated for $1 \mathrm{~h}$ only. In order to extend the same to multiple hour, a 24-h load profile as well as forecasted generation at bus 17 are shown in Figs. 11 and 12 for IEEE RTS-96 bus system, respectively. The two profiles represent the profiles of load and forecasted wind generation of transmission system of Elia (Belgian TSO) for November 3, 2014. However, it is scaled according to this test system. It is to be mentioned here that the forecast error of wind is assumed to be constant for all the hours, and is taken to be of the same value as stated previously. No contingency has been taken into account in this case.

Fig. 13 shows the total system operational confidence for $24 \mathrm{~h}$. Coordinated control of the PFCs helps in better system management, which is evident from the figure that more than $89 \%$ operational confidence (constrained at hour 19) can be achieved in this case with the coordinated control of both the PSTs. However, this involves changing the tap positions each hour (Fig. 14), thereby leading to operator intervention each hour.

In reality, the operators in the control room of the TSOs in Europe do not appreciate changing the control settings of the PFCs every hour, as it leads to increased wear and tear of the equipment. In order to overcome the above-said problem, the

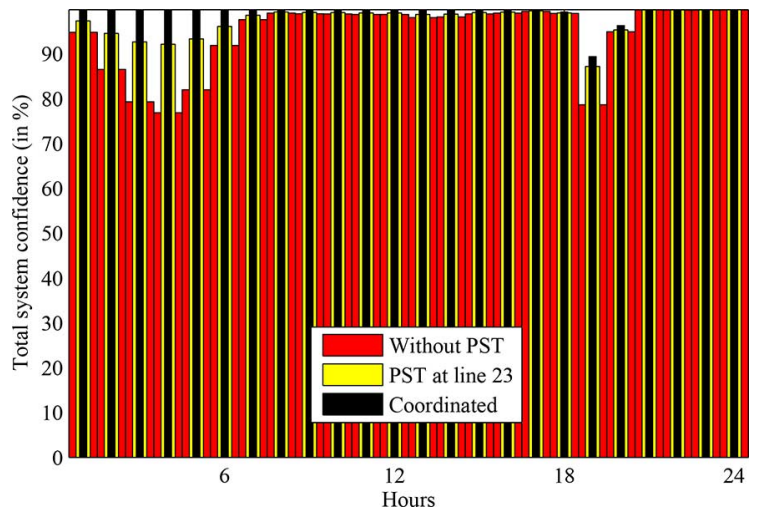

Fig. 13. Total system confidence.

operator can choose to have an $\epsilon$ probability of violation for the entire system, either for all of the hours or as required. This significantly eliminates the operator intervention each hour by keeping at a particular optimal point guaranteeing $1-\epsilon$ total system confidence for the concerned hours.

In order to achieve it, the optimization problem (14)-(23) needs some modifications. The following constraint needs to be added in order to force the system to be $1-\epsilon$ confident:

$$
\prod_{N}\left[\phi\left(z_{1-\epsilon_{R}}\right)-\phi\left(z_{1-\epsilon_{L}}\right)\right] \geq 1-\epsilon .
$$

\section{A. Problem Re-Formulation}

The optimization problem (14)-(23) can be re-formulated as

$$
\min _{u_{P F C}} \sum_{t=1}^{N_{h}-1} \sum_{|T|} \gamma_{t}
$$

subject to

$$
\begin{aligned}
& F_{t}^{\star}=\left(\nabla_{P} F\right)_{t} \cdot\left(P_{G}+P_{W}^{\star}-D\right)_{t} \quad \forall t \\
& F_{c, t}^{\star}=\left(\nabla_{P} F\right)_{c, t} \cdot\left(P_{G}+P_{W}^{\star}-D\right)_{t} \quad \forall c, t \\
& F_{t}^{\star}+z_{1-\epsilon_{R} t} \sqrt{\left(\nabla_{P} F\right)_{t}^{2} \cdot \sigma_{W t}^{2}} \\
& \quad+\left(\nabla_{u_{P F C}} F\right)_{t} \cdot u_{P F C_{t}} \leq \bar{F}_{t} \quad \forall t \\
& F_{t}^{\star}+z_{1-\epsilon_{L} t} \sqrt{\left(\nabla_{P} F\right)_{t}^{2} \cdot \sigma_{W t}^{2}} \quad \forall \\
& \quad+\left(\nabla_{u_{P F C}} F\right)_{t} \cdot u_{P F C_{t}} \geq \underline{F}_{t} \quad \forall t \\
& F_{c, t}^{\star}+\left(\nabla_{u_{P F C}} F\right)_{c, t} \cdot u_{P F C_{t}} \leq \bar{F}_{t} \quad \forall c, t \\
& F_{c, t}^{\star}+\left(\nabla_{u_{P F C}} F\right)_{c, t} \cdot u_{P F C_{t}} \geq \underline{F}_{t} \quad \forall c, t \\
& z_{1-\epsilon_{R t}} \geq 0 \quad \forall t \\
& z_{1-\epsilon_{L t}} \leq 0 \quad \forall t \\
& \underline{u}_{P F C} \leq u_{P F C_{t}} \leq \bar{u}_{P F C} \quad \forall t \\
& u_{P F C_{t}}-u_{P F C_{t+1}} \leq \gamma_{t} \quad \forall t \\
& u_{P F C_{t}}-u_{P F C_{t+1}} \geq-\gamma_{t} \quad \forall t \\
& \gamma_{t} \geq 0 \quad \forall t \\
& \prod_{N}\left[\phi\left(z_{1-\epsilon_{R t}}\right)-\phi\left(z_{1-\epsilon_{L t}}\right)\right] \geq 1-\epsilon \quad \forall t .
\end{aligned}
$$

Constraints (26)-(34) are same as that of constraints (15)-(23), but for each hour $t$. Constraints (35) and (36) make the change of the control settings of the PFCs as minimum as possible by 


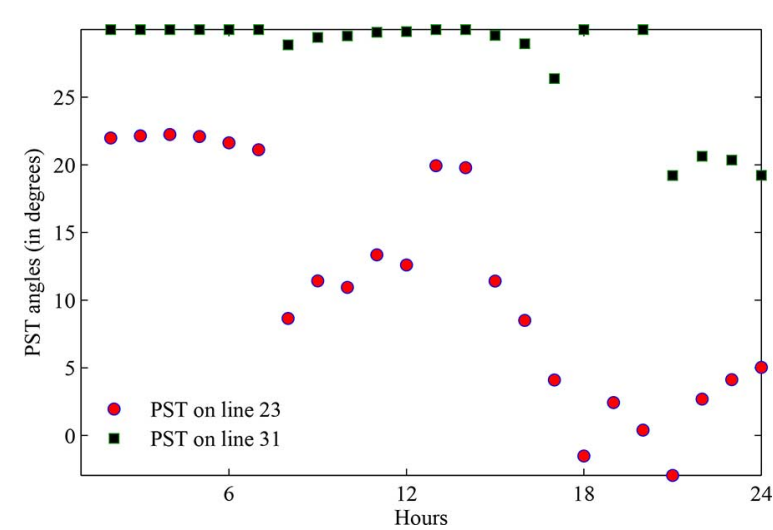

Fig. 14. PST angles.

the positive variable $\gamma \in \mathbb{R}^{|T|}$ [constraint (37)], which is minimized in the objective function over all the PFCs and the considered hours. Finally, constraint (38) represents the violation probability of the whole system to be at-most $\epsilon$.

\section{B. Simulation Results}

A close look at Fig. 13 reveals that at hour 19, a maximum total system confidence of $89 \%$ can be achieved with the coordinated control of the PSTs. More than $99 \%$ is achievable for the hours before that. Hence, the operator can either choose to have a maximum of $89 \%$ system confidence for all $24 \mathrm{~h}$ or $99 \%$ until hour 18 and $89 \%$ after that.

Fig. 15 shows the result of the above optimization problem (25)-(38). Fig. 15(a) shows the PST angles for a total system confidence of $89 \%$ for all the hours, whereas Fig. 15(b) shows the same for a total system confidence of $99 \%$ until hour 17 and $89 \%$ after that. It needs to be mentioned here that the total system confidence for hour 18 is chosen to be $89 \%$ instead of $99 \%$, as the operators prefer to already change taps $1 \mathrm{~h}$ before the constrained hour (hour 19 is constrained in this case) in order to have a significant time margin if complications arise.

Comparing Figs. 14 and 15 clearly reveals that less [one in Fig. 15(b)] or no [Fig. 15(a)]operator intervention is required. This is acceptable from an operator point of view.

\section{CONCLUSION}

A novel approach to include uncertainties in the day-ahead scheduling process of a power system is proposed in this paper. This approach quantifies the amount of achievable confidence and its improvement with the help of PFC devices, as the operation of these devices comes at a near zero cost to the TSOs. In order to address this, a framework is developed for stochastic optimization including PFC devices. The effect of coordination of multiple PFC devices present in the system is also demonstrated in this paper. A methodology considering operator concerns to minimize changes in control settings of the PFCs over multiple hour time-frame taking a predetermined total system operation confidence into account is also proposed in this paper.

The demonstrations are conducted on three test systems. The method is also validated on one of the test system using MC simulations. The results show that the proposed methodology helps the TSOs to determine the optimal control settings of their PFCs with a predefined certainty of operation. The proposed

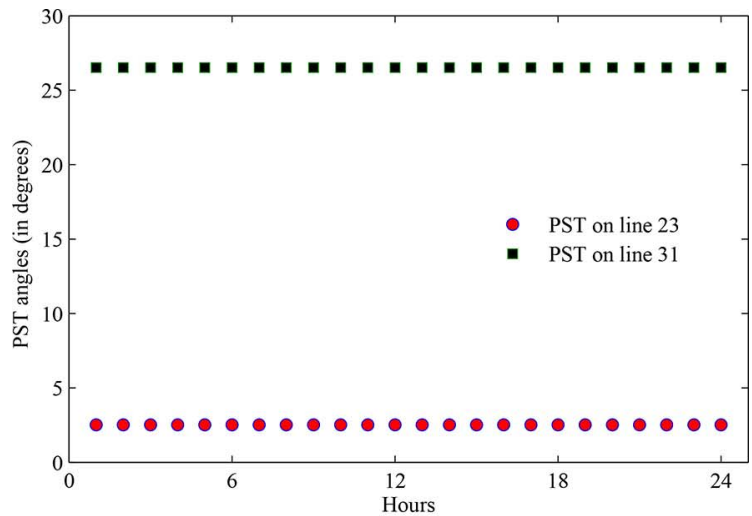

(a)

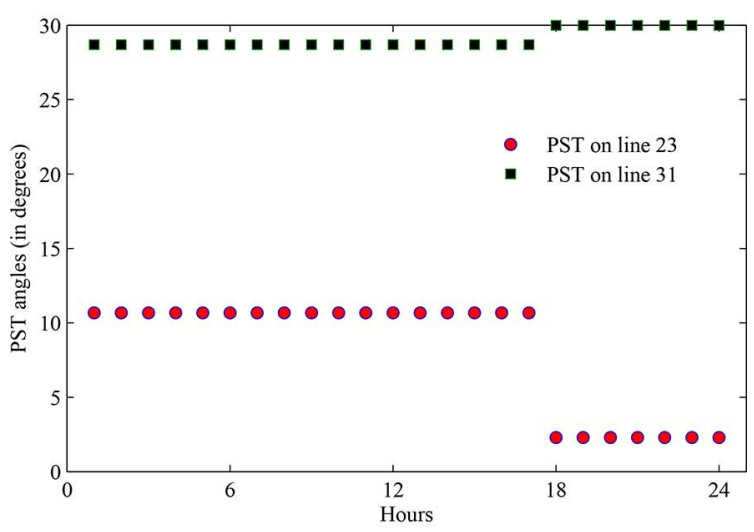

(b)

Fig. 15. PST angles. (a) Total system confidence of $89 \%(\epsilon=11 \%)$ for all the hours. (b) Total system confidence of $99 \%(\epsilon=1 \%)$ until hour 17 and $89 \%$ beyond.

methodology is therefore helpful for day-ahead scheduling of power systems taking uncertainties into account and minimize the system operation risks due to these uncertainties with the help of already installed PFCs in their systems.

\section{APPENDIX A}

Single Line Diagram of IEEE 14-Bus System

Fig. 16 shows the IEEE 14-bus system [35].

APPENDIX B

SiNGLE LiNE DiAgRAM OF IEEE RTS-96 SYSTEM

Fig. 17 shows the IEEE RTS-96 system [36].

\section{APPENDIX C MILP PROBLEM}

The formulation of the MILP problem is as follows:

$$
\min _{u_{P F C}} \sum_{s} z_{s}
$$

subject to 


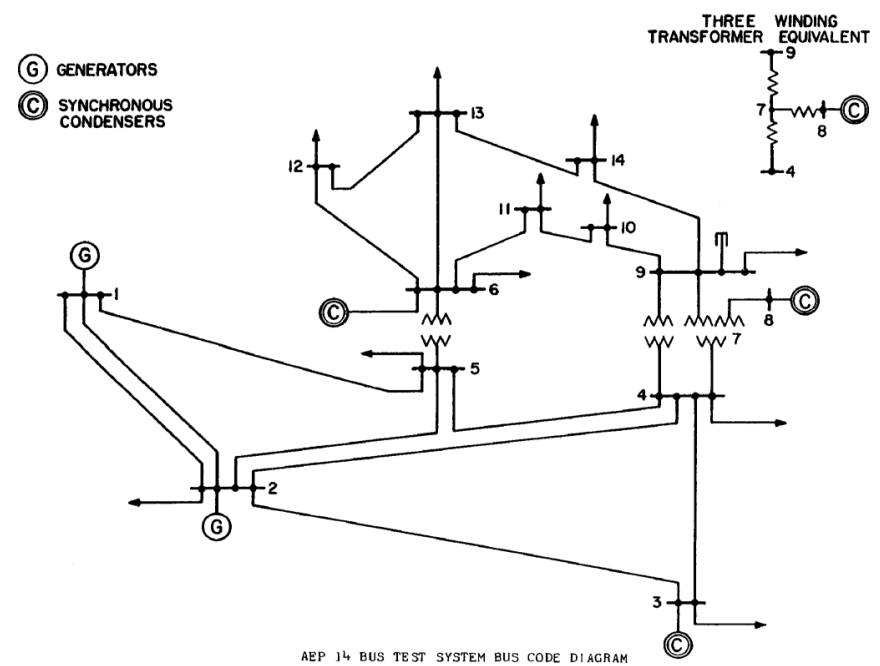

Fig. 16. IEEE 14-bus system [35].

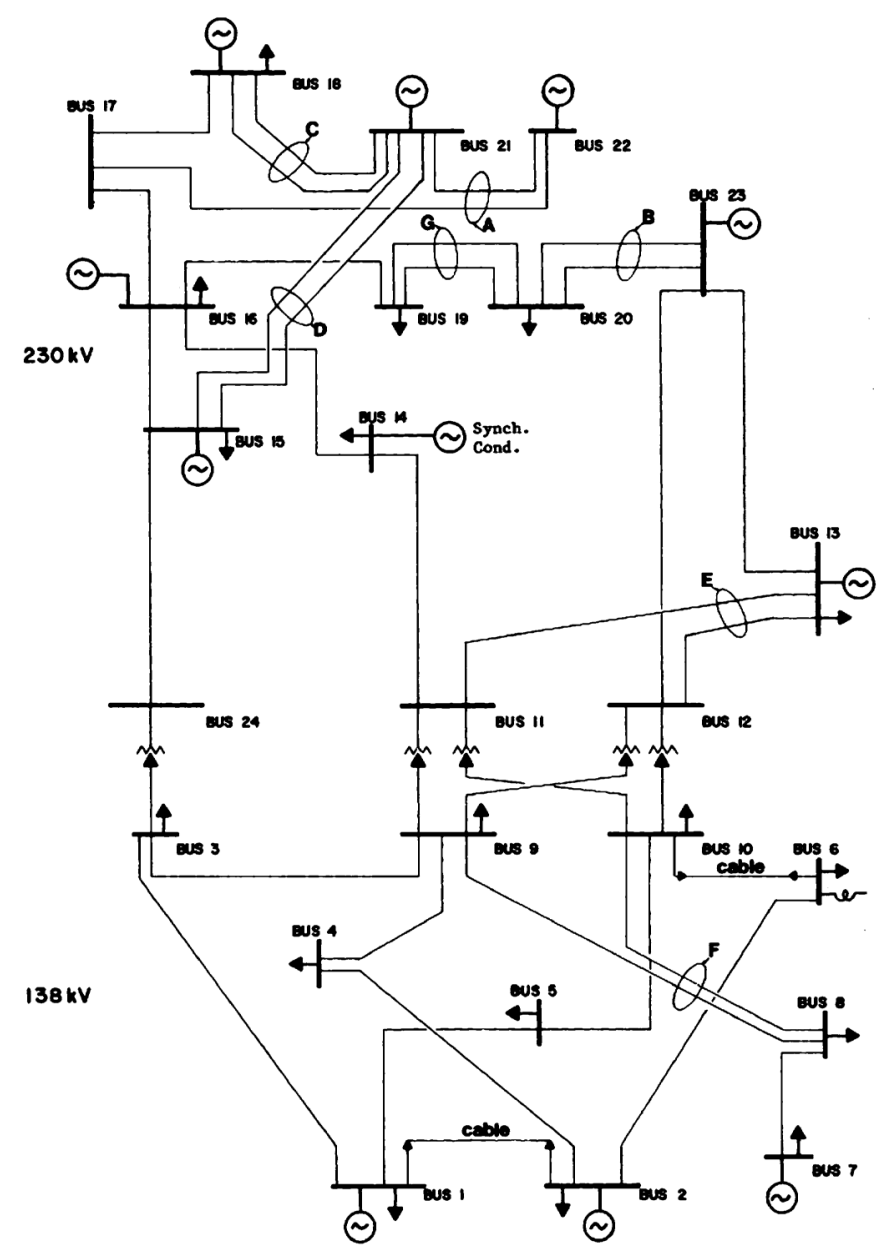

Figure 1 - IRER Reliability Test System

Fig. 17. IEEE RTS-96 system [36].

$$
\begin{aligned}
& F_{s}=F_{s}^{\star}+\left(\nabla_{u_{P F C}} F\right)_{s} \cdot u_{P F C} \\
& F_{s} \leq\left(1-z_{s}\right) \bar{F}+M z_{s} \\
& F_{s} \geq\left(1-z_{s}\right) \underline{F}-M z_{s} \\
& \underline{u}_{P F C} \leq u_{P F C} \leq \bar{u}_{P F C} \\
& z_{s} \in\{0,1\} \quad \forall s .
\end{aligned}
$$

Constraint (40) represents the line flows for each scenario $s$, in which $F_{s}$ and $F_{s}^{\star}$ are the flows and the reference flows, respectively, for scenario $s$. Constraints (41) and (42) are the limits on line flows for each scenario $s$, where $\bar{F}$ and $\underline{F}$ are the upper and lower limits on line flows, respectively. In these constraints, $M$ is a sufficiently large number. When $z_{s}=0$ (a binary variable for scenario $s$, represented by (44)), the term containing $M$ becomes 0 in (41) and (42). Both the constraints together ensure that all the flows are within their respective limits with the help of PST for that particular scenario $s$. When $z_{s}=1$, the term containing $M$ dominates the other terms in (41) and (42) and hence the flows do not respect their respective limits for that particular scenario $s$. Constraint (43) is same as that of constraint (23). Finally the idea is to minimize the number of total scenarios that violate the line flow constraints, and is represented by (39).

\section{REFERENCES}

[1] P. Panciatici, Y. Hassaine, L. Fliscounakis, M. Platbrood, J. OrtegaVazquez, Martinez-Ramoz, and L. Wehenkel, "Security management under uncertainty: From day-ahead planning to intraday operation," in Proc. 2010 iREP Symp. Bulk Power System Dynamics and Control (iREP) - VIII (iREP), Aug. 2010, pp. 1-8.

[2] F. Capitanescu, S. Fliscounakis, P. Panciatici, and L. Wehenkel, "Cautious operation planning under uncertainties," IEEE Trans. Power Syst., vol. 27, no. 4, pp. 1859-1869, Nov. 2012.

[3] S. Fliscounakis, P. Panciatici, F. Capitanescu, and L. Wehenkel, "Contingency ranking with respect to overloads in very large power systems taking into account uncertainty, preventive, and corrective actions," IEEE Trans. Power Syst., vol. 28, no. 4, pp. 4909-4917, Nov. 2013.

[4] D. S. Kirschen and D. Jayaweera, "Comparison of risk-based and deterministic security assessments," IET Gener., Transm., Distrib., vol. 1, no. 4, pp. 527-533, 2007.

[5] A. Monticelli, M. V. F. Pereira, and S. Granville, "Security-constrained optimal power flow with post-contingency corrective rescheduling," IEEE Trans. Power Syst., vol. 2, no. 1, pp. 175-180, Feb. 1987.

[6] D. Phan and J. Kalagnanam, "Some efficient optimization methods for solving the security-constrained optimal power flow problem," IEEE Trans. Power Syst., vol. 29, no. 2, pp. 863-872, Mar. 2014.

[7] F. Capitanescu and L. Wehenkel, "A new iterative approach to the corrective security-constrained optimal power flow problem," IEEE Trans. Power Syst., vol. 23, no. 4, pp. 1533-1541, Nov. 2008.

[8] F. Bouffard and A. J. Ardakani, "Identification of umbrella constraints in DC-based security-constrained optimal power flow," IEEE Trans. Power Syst., vol. 28, no. 4, pp. 3924-3934, Nov. 2013.

[9] L. Platbrood, F. Capitanescu, C. Merckx, H. Crisciu, and L. Wehenkel, "A generic approach for solving nonlinear-discrete security-constrained optimal power flow problems in large-scale systems," IEEE Trans. Power Syst., vol. 29, no. 3, pp. 1194-1203, May 2014.

[10] Q. Jiang and K. Xu, "A novel iterative contingency filtering approach to corrective security-constrained optimal power flow," IEEE Trans. Power Syst., vol. 29, no. 3, pp. 1099-1109, May 2014.

[11] F. Capitanescu, M. Glavic, D. Ernst, and L. Wehenkel, "Contingency filtering techniques for preventive security-constrained optimal power flow," IEEE Trans. Power Syst., vol. 22, no. 4, pp. 1690-1697, Nov. 2007.

[12] B. Borkowska, "Probabilistic load flow," IEEE Trans. Power App. Syste., vol. PAS-93, no. 3, pp. 752-759, May 1974.

[13] J. Usaola, "Probabilistic load flow in systems with wind generation," IET Gener., Transm., Distrib., vol. 3, no. 12, pp. 1031-1041, Dec. 2009.

[14] Z. Hu and X. Wang, "A probabilistic load flow method considering branch outages," IEEE Trans. Power Syst., vol. 21, no. 2, pp. 507-514, May 2006.

[15] M. Vrakopoulou, K. Margellos, J. Lygeros, and G. Andersson, "Probabilistic guarantees for the $\mathrm{N}-1$ security of systems with wind power generation," in Proc. PMAPS 2012, Istanbul, Turkey, 2012.

[16] M. Vrakopoulou, M. Katsampani, K. Margellos, J. Lygeros, and G. Andersson, "Probabilistic security-constrained AC optimal power flow," in Proc. IEEE Powertech Grenoble, Jun. 16-20, 2013, pp. 1-6.

[17] G. L. Viviani and G. T. Heydt, "Stochastic optimal energy dispatch," IEEE Trans. Power App. Syst, vol. PAS-100, no. 7, pp. 3221-3228, Jul. 1981. 
[18] F. Bouffard and F. D. Galiana, "Stochastic security for operations planning with significant wind power generation," IEEE Trans. Power Syst., vol. 23, no. 2, pp. 306-316, May 2008.

[19] F. Bouffard and F. D. Galiana, "An electricity market with a probabilistic spinning reserve criterion," IEEE Trans. Power Syst., vol. 19, no. 1, pp. 300-307, Feb. 2004.

[20] F. Bouffard, F. D. Galiana, and A. J. Conejo, "Market-clearing with stochastic security-Part I: Formulation," IEEE Trans. Power Syst., vol. 20 , no. 4, pp. 1818-1826, Nov. 2005.

[21] F. Bouffard, F. D. Galiana, and A. J. Conejo, "Market-clearing with stochastic security-Part II: Case studies," IEEE Trans. Power Syst., vol. 20, no. 4, pp. 1827-1835, Nov. 2005.

[22] Y. Wang, Q. Xia, and C. Kang, "A novel security stochastic unit commitment for wind-thermal system operation," in Proc. 4th Int. Conf. Electric Utility Deregulation and Restructuring and Power Technologies (DRPT), Jul. 6-9, 2011, pp. 386-393.

[23] Y. Li and J. D. McCalley, "Risk-based optimal power flow and system operation state," in Proc. IEEE Power \& Energy Soc. General Meeting, Jul. 26-30, 2009, pp. 1-6, 2009.

[24] H. Yu and W. Rosenhart, "An optimal power flow algorithm to achieve robust operation considering load and renewable generation uncertainties," IEEE Trans. Power Syst., vol. 27, no. 4, pp. 1808-1817, Nov. 2012.

[25] D. Van Hertem, J. Rimez, and R. Belmans, "Power flow controlling devices as a smart and independent grid investment for flexible grid operations: Belgian case study," IEEE Trans. Smart Grid, vol. 4, no. 3, pp. 1656-1664, Sep. 2013.

[26] M. Vrakopoulou, S. Chatzivasileiadis, and G. Andersson, "Probabilistic security-constrained optimal power flow including the controllability of HVDC lines," in Proc. 4th IEEE/PES Innovative Smart Grid Technologies Europe (ISGT Europe), Oct. 6-9, 2013, pp. $1-5,2013$.

[27] M. Vrakopoulou, S. Chatzivasileiadis, E. Iggland, M. Imhof, T. Krause, O. Makela, J. L. Mathieu, L. Roald, R. Wiget, and G. Andersson, "A unified analysis of security-constrained OPF formulations considering uncertainty, risk, and controllability in single and multi-area systems," in IProc. REP Symp.-Bulk Power Systems Dynamics and Control-IX, Rethymnon, Greece, Aug. 25-30, 2013.

[28] Q. Wang, J. McCalley, T. Zheng, and E. Litvinov, "A computational strategy to solve preventive risk-based security-constrained OPF," IEEE Trans. Power Syst., vol. 28, no. 2, pp. 1666-1675, May 2013.

[29] D. L. Olson and S. R. Swenseth, "A linear approximation for chance-constrained programming," J. Oper. Res. Soc., vol. 38, no. 3, pp. 261-267, Mar. 1987.

[30] Y. Seppälä, "A chance-constrained programming algorithm," BIT Numer. Math., vol. 12, no. 3, pp. 376-399, 1972.

[31] A. Charnes and W. W. Cooper, "Chance-constrained programming," Manage. Sci., vol. 6, no. 1, pp. 73-79, Oct. 1959.

[32] M. Campi, S. Garatti, and M. Prandini, "The scenario approach for systems and control design," Annu. Rev. Control, vol. 33, no. 2, pp. 149-157, 2009.

[33] U. Focken, M. Lange, K. Mnnich, H. P. Waldl, H. G. Beyer, and A. Luig, "Short-term prediction of the aggregated power output of wind farms-A statistical analysis of the reduction of the prediction error by spatial smoothing effects," J. Wind Eng. Ind. Aerodynam., vol. 90, no. 3, pp. 231-246, 2002.

[34] L. Roald, F. Oldewurtel, T. Krause, and G. Andersson, "Analytical reformulation of security constrained optimal power flow with probabilistic constraints," in Proc. IEEE Powertech Conf., Grenoble, France, Jun. 16-20, 2013, pp. 1-6.

[35] P. M. Anderson and A. A. Fouad, Power System Control and Stability. Piscataway, NJ, USA: IEEE Press/Wiley, 1994.

[36] Reliability Test System Task Force of the Application of Probability Methods Subcommittee, "The IEEE reliability test system-1996," IEEE Trans. Power Syst., vol. 14, no. 3, pp. 1010-1020, Aug. 1999.

[37] R. D. Zimmerman, C. E. Murillo-Sanchez, and R. J. Thomas, "Matpower: Steady-state operations, planning and analysis tools for power systems research and education," IEEE Trans. Power Syst., vol. 26, no. 1, pp. 12-19, Feb. 2011.
[38] GAMS Development Corporation, GAMS: The Solver Manuals. Washington, DC, USA, 2005.

[39] P. Guha Thakurta, J. Maeght, R. Belmans, and D. Van Hertem, "Increasing transmission grid flexibility by TSO coordination to integrate more wind energy sources while maintaining system security," IEEE Trans. Sustain. Energy, to be published.

[40] S. Boyd and L. Vandenberghe, Convex Optimization. Cambridge, U.K.: Cambridge Univ. Press, 2004.

[41] A. Papoulis, Probability, Random Variables, and Stochastic Processes. Boston, MA, USA: McGraw-Hill, 1991.

[42] J. R. Birge and F. Louveaux, Introduction to Stochastic Programming. New York, NY, USA: Springer, 1997.

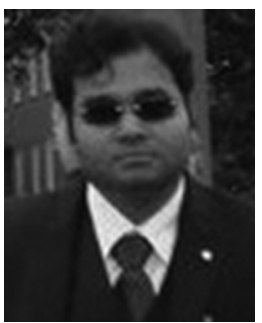

Priyanko Guha Thakurta (S'10-M'14) received the M.Sc. degree in electrical engineering from KTH, Stockholm, Sweden, in 2009. Since 2010, he has been pursuing the Ph.D. degree at KU Leuven, Leuven, Belgium.

His special fields of interest are effects of FACTS devices and coordination of power flow controllers in power systems.

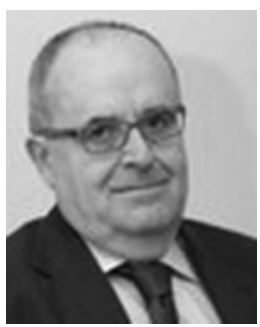

Ronnie Belmans (S'77-M'84-SM'89-F'05) received the M.Sc. degree in electrical engineering in 1979 and the Ph.D. degree in 1984, both from KU Leuven, Leuven, Belgium. In 1989 he added a Special Doctorate from the KULeuven and in 1993 a "Habilitierung", from the RWTH, Aachen, Germany.

Currently, he is a full Professor at KU Leuven, teaching techno-economical aspects of power systems, electrical energy, and regulatory affairs, among others. His research interests include smart grids, security of energy supply, and the techno-economic aspects of the liberalization of the electricity market. Within Belgium, he is vice president of the KU Leuven Energy Institute as well as cofounder and $\mathrm{CEO}$ of EnergyVille, a research collaboration in Genk specializing in energy in smart cities and buildings, in cooperation with VITO and IMEC. On a global scale, he is executive director of the Global Smart Grids Federation (GSGF). $\mathrm{He}$ is also honorary chairman of the board of directors of ELIA, the Belgian transmission system operator.

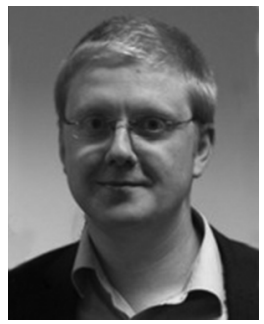

Dirk Van Hertem (S'02-M'08-SM'09) was born in 1979 in Neerpelt, Belgium. He received the M.Eng. degree in 2001 from the KHK, Geel, Belgium, the M.Sc. degree in electrical engineering from the $\mathrm{KU}$ Leuven, Leuven, Belgium, in 2003, and the Ph.D. degree in 2009 from the KU Leuven.

In 2010, he was a member of the EPS group at the Royal Institute of Technology, Stockholm, Sweden, where he was the program manager for controllable power systems for the $\mathrm{EKC}^{2}$ competence center at KTH. Since spring 2011, he has been back at the University of Leuven, where he is an Assistant Professor in the ELECTA group. His special fields of interest are power system operation and control in systems with FACTS and HVDC and building the transmission system of the future, including offshore grids and the supergrid concept.

Dr. Van Hertem is an active member of both IEEE (PES and IAS) and Cigré. 